\title{
Observation and Analysis of RW Doradus
}

\author{
B. F. Marino ${ }^{\mathrm{A}}$, W. S. G. Walker $^{\mathrm{B}}$, C. Bembrick ${ }^{\mathrm{C}}$, and E. Budding ${ }^{\mathrm{D}, \mathrm{E}}$ \\ A Auckland Observatory, PO Box 24180 Royal Oak, Auckland 1345, New Zealand \\ ${ }^{B}$ Wharemaru Observatory, Awanui 0552, Kaitaia, New Zealand \\ ${ }^{C}$ Mt. Tarana Observatory, PO Box 1537, Bathurst, NSW 2795, Australia \\ D Carter Observatory, PO Box 2909, Wellington, New Zealand \& Physics Dept., \\ 18th March University of Canakkale, Turkey

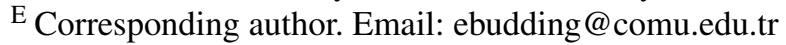

Received 2007 March 07, accepted 2007 September 27

\begin{abstract}
Photometry of the short-period ( $P \sim 0.285 \mathrm{~d})$ low-mass $\left(M_{1} \sim 0.67, M_{2} \sim 0.48 \mathrm{M}_{\odot}\right)$ W UMa-type eclipsing binary RW Dor, mainly involving a good series of observations in 1987-88, using standard $B$ and $V$ filters at the Auckland Observatory, is examined. Analysis via light curve fittings alone permits both transit (near main sequence) and occultation (W-type contact binary) solutions. Consideration of proximity or other effects on the relatively small number of measured radial velocities gives rise to a more detailed review of the alternatives, though the (W-type) configuration determined by Hilditch, Hill \& Bell (1992) is still confirmed. Further implications of this configuration are examined, given an apparent general trend to period decrease $\left(\triangle P / P \sim 6 \times 10^{-11}\right)$, and some light curve asymmetry. This is consistent with the initially low-mass companion, Case-B scenario of Budding (1984). The evidence does suggest irregularity of the period variation however, and further detailed surveillance, including more spectroscopy, is desirable.
\end{abstract}

Keywords: stars: close binaries — stars: late-type — stars: individual: RW Dor — general: contact binaries techniques: photometric light curve analysis

\section{Introduction}

The short-period eclipsing binary nature of RW Dor (= HDE 269320, HIP 24763) was discovered by Hertzsprung (1928) on plates taken at Johannesburg. Hertzsprung produced a light curve from careful examination of a number of such plates using a Schilt microphotometer. The two minima are appreciably different in depth, though the continuous light variation and short ( $\sim 7 \mathrm{~h}$ ) period have been used to put RW Dor among the W UMa-type stars. The period is one of the shortest known for the more usual types of eclipsing binaries. The K5 spectral classification (McLaughlin 1927) is in keeping with low-mass, small stars. Although in the general field of the Large Magellanic Cloud (LMC), with an out-of-eclipse magnitude of $V \sim 10.8$, it can easily be deduced that RW Dor could not possibly be a member of that galaxy. In fact, the Hipparcos parallax (ESA 1997) corresponds to a distance of $112 \pm \sim 25 \mathrm{pc}$.

Full photometric coverage of the variable was reported by Kaluzny \& Caillault (1989) and Marton, Grieco \& Sistero (1989), together with summaries of previous work. These mention variability of the light curve shape as well as the significant difference in eclipse depths. Kaluzny \& Caillault produced $U B V R I_{c}$ light curves from five nights of observations in 1986; while the $U B V$ light curves of Marton et al. are spread over 29 nights between 1979 and 1982. Using the program of Wilson \& Devinney (1971), both these papers studied a wide range of overcontact models that might match the data, of both 'A' (photometric primary is more massive) and ' $\mathrm{W}$ ' (photometric primary is less massive) types. These ambiguities point significantly to the essential indeterminacy of photometric evidence, when taken in isolation, in analysing W UMatype binary stars. We will examine this point by our own separate analyses in what follows.

Such uncertainties may well have motivated Hilditch et al. (1992) to carry out spectroscopic observations using the $3.9 \mathrm{~m}$ telescope of the Anglo-Australian Observatory in 1990-91. The RGO cassegrain spectrograph was used, with a dispersion of $33 \mathrm{~A} / \mathrm{mm}$ and a 1200 lines per $\mathrm{mm}$ grating. Exposures were typically $10 \mathrm{~min}$ in duration, though sometimes observed through clouds. Although the radial velocity coverage of Hilditch et al. appears rather sparse and scattered, the authors concluded that the binary was of ' $\mathrm{W}$ ' type, thus conforming to the more usual physical situation found for cool W UMa-type binary systems (Yamasaki 1975; Budding 1981).

\section{Observations}

The star was observed at the Auckland Observatory (AO) on eight nights in 1987-88 (Table 1) using the Edith Winstone Blackwell $50 \mathrm{~cm}$ telescope and the observatory photometer, equipped with an EMI 9502 tube and the Griffiths photon counting system. The main comparison star was HD 35293; $V=9.21, B-V=0.25$ (SIMBAD). The check star was HD 35230; $V=7.59, B-V=0.86$ 
Table 1. Observing log for RW Dor photometry at Auckland Observatory, 1987-88

\begin{tabular}{|c|c|}
\hline Date & Comment \\
\hline 1987 Dec 20 & Conditions fair, few pts \\
\hline 1988 Jan 10 & Ok to UT 09 40, and from UT 1144 on \\
\hline 1988 Jan 11 & $\begin{array}{l}\text { Dark current problems UT } 1112 \text { to } 1200 \text {, } \\
\text { good before and after }\end{array}$ \\
\hline 1988 Jan 18 & Data after UT 1012 suspect, not used \\
\hline 1988 Jan 21 & All data good \\
\hline 1988 Jan 22 & All data good \\
\hline 1988 Jan 27 & All data good, but some interruptions \\
\hline 1988 Feb 19 & All data good \\
\hline
\end{tabular}

(SIMBAD). According to our reduced measurements, HD 35230 has $V=7.60 \pm 0.010$ and $B-V=0.86 \pm 0.013$, where the errors are s.d. values.

Altogether, about 250 individual measures were made in each of $U, B \& V$ filters. The $U$ data are very noisy, however, and have been dropped from the present study. The $B$ and $V$ light curves resemble those of the W UMa type, with a good phase coverage. These data were later prepared and analysed at Carter Observatory. They are available (in ASCII format) as an Accessory Publication. The photometric ephemeris

$$
\text { Min } I=J D 2430938.6017+0.285463812 E
$$

Kholopov (1985) was used for the phasing.

Efforts were made in the early 1990s to use the $1.8-\mathrm{m}$ telescope and Cassegrain spectrograph of the Mt Stromlo Observatory to obtain radial velocities to supplement this photometry (Banks, Sullivan \& Budding 1990). The combination of light-grasp with appropriate resolution, faintness of source and usable exposure times, given the $7 \mathrm{~h}$ period has, however, rendered the $\mathrm{S} / \mathrm{N}$ of these spectrograms unsuitable for clear radial velocities. ${ }^{1}$ The radial velocities of Hilditch et al. (1992) were published meanwhile, enabling masses to be derived.

\section{Analysis and the Contact Question}

Budding (1981) queried the inference that all photometrically classified W UMa-type binaries have a common envelope physical configuration. Some such binaries might actually be unevolved dwarfs in very close proximity. The question becomes more pertinent, the more different are the eclipse depths. The incidence of detached but very close pairs is, in any case, relevant to theories of the evolution of W UMa-type binaries, and has been investigated in a number of observational studies (Hilditch 1989; Rucinski \& Lu 1999). RW Dor appears as an interesting test case in interpreting this type of light curve.

Light-curve fitting experiments usually start with a suitable set of preliminary estimates that can be taken from inspection of the light curves (Budding \& Demircan,

\footnotetext{
${ }^{1}$ The original spectrographic data are available, on request, from Dr D. J. Sullivan, School of Chemical and Physical Sciences, VUW, Wellington, New Zealand.
}

Table 2. Preliminary light-curve fitting results for $\mathbf{R W}$ Dor

\begin{tabular}{lc}
\hline Parameters & Values \\
\hline$T_{\mathrm{h}}(\mathrm{K})$ & 4800 \\
$T_{\mathrm{c}}(\mathrm{K})$ & 4100 \\
$M_{2} / M_{1}$ & 0.6 \\
$L_{1}-\mathrm{B}$ & $0.720(5)$ \\
$L_{1}-\mathrm{V}$ & $0.632(5)$ \\
$L_{2}-\mathrm{B}$ & $0.280(5)$ \\
$L_{2}-\mathrm{V}$ & $0.368(5)$ \\
$r_{1}(\mathrm{mean})$ & $0.407(2)$ \\
$r_{2}(\mathrm{mean})$ & $0.354(2)$ \\
$i(\mathrm{deg})$ & $77.1(0.3)$ \\
$\Delta \phi_{0}(\mathrm{deg})$ & 3.0 \\
$\Delta l$ & 0.015 \\
\hline
\end{tabular}

2007). The $B-V$ colour at secondary minimum, when the binary is least red i.e. the light is dominated by the primary, is 0.94 . This would be consistent with the K3V type reported in the SIMBAD database, although Kholopov (1985) kept the older K5 classification. The primary temperature was then set at $4800 \mathrm{~K}$. The relative luminosities in the two colours allow a preliminary estimate of the difference of colour $\Delta(B-V)$ as about 0.44 , making the secondary (the cooler star, here with suffix 2) a late K-type dwarf of temperature around $4100 \mathrm{~K}$. These temperatures were adopted for preliminary curve-fittings. A trial Main Sequence (MS)-like model would then consist of two stars of masses about 0.66 and $0.44 \mathrm{M}_{\odot}$. The period $(0.285463812 \mathrm{~d})$, taken together with Kepler's Third Law and the foregoing masses implies a separation of about $1.88 \mathrm{R}_{\odot}$. A corresponding parameter set resulting from such estimates put into an 'Information Limit Optimization Technique' (ILOT) curve-fitter is given in Table 2. The name ILOT was introduced in Banks \& Budding (1990), but a thorough discussion of the subject is given in Budding \& Demircan (2007). The corresponding curve-fit is shown in Figure 1. Symbols have their usual meanings (e.g. Budding \& Demircan 2007). Note that $\Delta l$ corresponds to an adopted measurement accuracy (as a fraction of the normalized light level), whose value allows the corresponding reduced $\chi^{2}$ measure of the goodness of fit to accord with accidental errors of measurement.

The formal errors, indicated in brackets, should be regarded circumspectly. The numbers given correspond to mutual independence in effect on the light curve fitting. When inter-correlation effects are taken into account a five-parameter solution remains formally determinate, but the errors increase: particularly for the fractional luminosities (the eclipses being partial), where interdependent error estimates can increase by a factor $\sim 5$. The wider implications of such procedures should be kept in mind, even with a more complete analysis of the determinacy Hessian; since, of course, this mathematical 'solution' may well be entirely wrong physically (as will be seen shortly).

Although the secondary would be somewhat large for its mass, the configuration is not far from a pair of very 

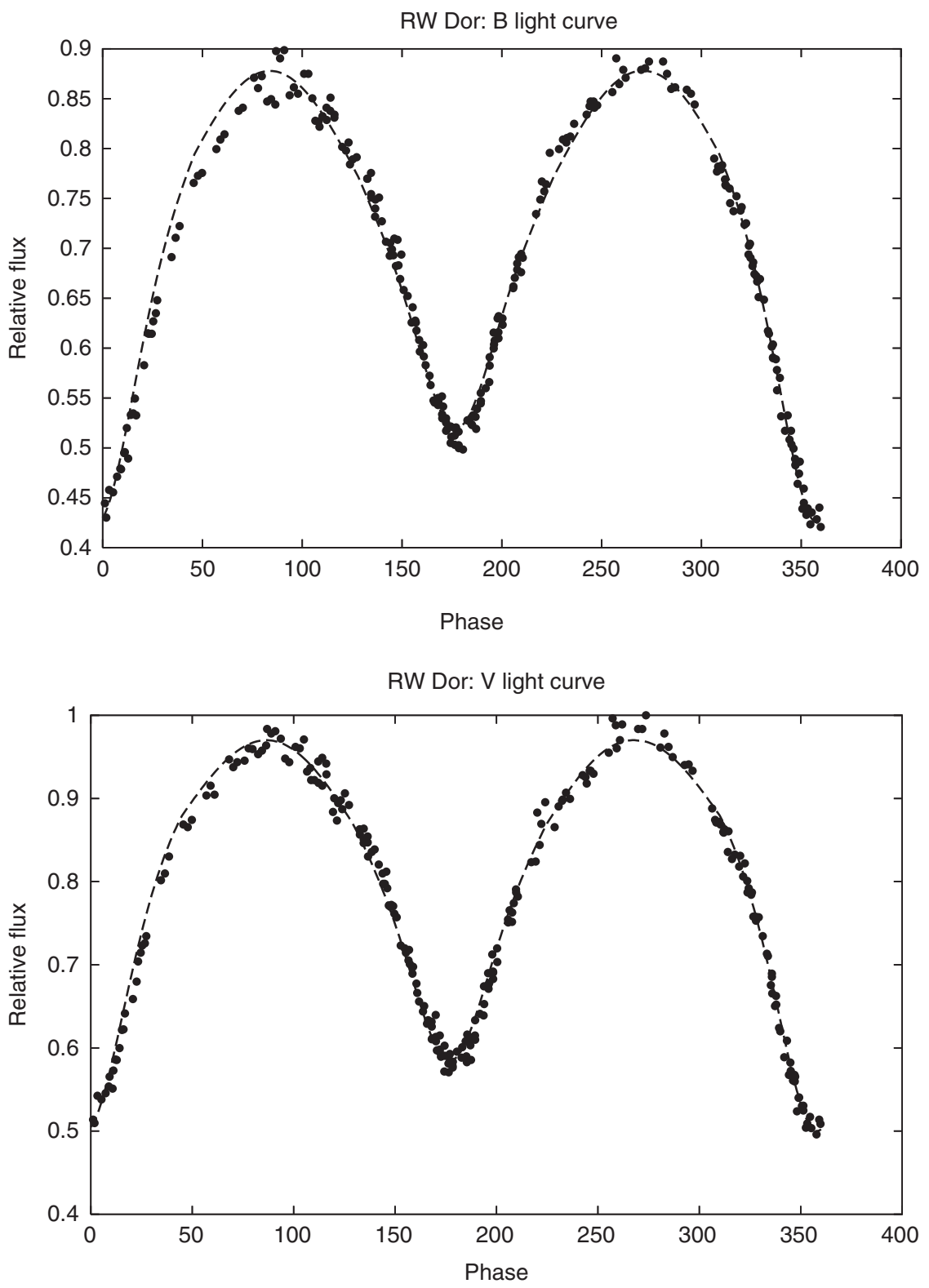

Figure 1 Light curves of RW Dor and their transit-model fitting according to the parameters of Table 2.

close MS-type dwarfs (e.g. Budding \& Demircan 2007, Table 3.6). It is worth noticing this configuration, consistent with the photometry alone, on methodological grounds, although it is denied by the radial velocities of Hilditch et al. (1992). In view of the critical nature of the radial velocity fitting of Hilditch et al. we decided to re-examine those data. For this we have applied the treatment of proximity effects on the radial velocities (obviously of significance in the case of a contact, or near-contact, binary) discussed by Kopal (1979). Kopal's treatment follows classical methods of solving the Poisson Equation for problems involving gravitational forces, i.e. by the use of spherical harmonics in progressive approximation series. In its generality for the mass distribution and rotation, this is more physically flexible than the popular 'Roche-lobe' approximations.
The results of such fittings are shown in Figure 2 and corresponding parameters listed in Table 3. Again, symbols have normal usage. $\Delta v$ similarly corresponds to the adopted measurement accuracy (in $\mathrm{km} \mathrm{s}^{-1}$ ), whose value, if due only to accidental errors of measurement, allows a reasonable probability to the model underlying the fitting. At the suggestion of a referee, we have added another 8 points to the radial velocities, coming from Duerbeck \& Rucinski (2007). These extra points, although not too concordant with expectation, do not significantly change the previous results. Formal errors for the dependent separations $\left(a_{1,2}\right)$ and masses $\left(m_{1,2}\right)$, resulting from the radial-velocity curve fits are listed in the brackets. The velocity amplitudes are formally obtained to within $2-3 \%$ of their values, but the spread of velocities given by the different authors (using different methods) may be a better 


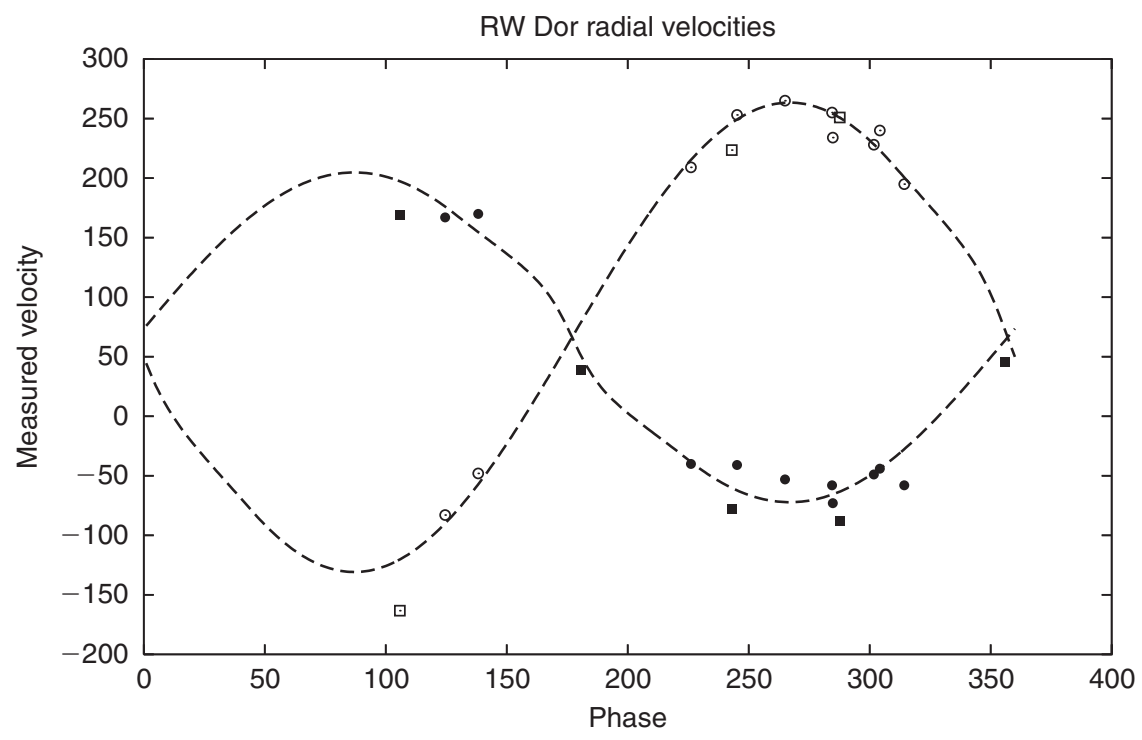

Figure 2 Radial-velocity curves of Hilditch et al. (1992, circles) were first fitted using the Kopal (1979) linearization for proximity effects and rotational effects in eclipse. The more recent measures of Duerbeck \& Rucinski (2006, boxes) were then added and a fit to the combined data is shown. (Full symbols for primary, empty for secondary.)

Table 3. Radial-velocity fitting for the data of Hilditch et al. (1992) and Duerbeck \& Rucinski (2006)

\begin{tabular}{lcccc}
\hline Parameters & \multicolumn{3}{c}{ Values } \\
& $\begin{array}{c}\text { This paper } \\
\text { I }\end{array}$ & $\begin{array}{c}\text { Hilditch et al. } \\
(1992)\end{array}$ & $\begin{array}{c}\text { Duerbeck \& Rucinski } \\
(2006)\end{array}$ & $\begin{array}{c}\text { This paper } \\
\text { II }\end{array}$ \\
\hline$K_{1}\left(\mathrm{~km} \mathrm{~s}^{-1}\right)$ & 135.6 & 130.5 & 134.4 & 138.5 \\
$K_{2}\left(\mathrm{~km} \mathrm{~s}^{-1}\right)$ & 192.6 & 191.5 & 224.2 & 197.1 \\
$V_{\gamma}\left(\mathrm{km} \mathrm{s}^{-1}\right)$ & 66.3 & 66.5 & 41.1 & 66.3 \\
$a_{1} \mathrm{R}_{\odot}$ & $0.79(3)$ & 0.76 & 0.78 & $0.80(4)$ \\
$a_{2} \mathrm{R}_{\odot}$ & $1.12(2)$ & 1.11 & 1.30 & $1.14(4)$ \\
$M_{1} \mathrm{M}_{\odot}$ & $0.67(9)$ & 0.64 & 0.87 & $0.71(12)$ \\
$M_{2} \mathrm{M}_{\odot}$ & $0.48(5)$ & 0.43 & 0.61 & $0.49(11)$ \\
$\Delta v$ & 10.3 & & & \\
\hline
\end{tabular}

reflection of the real errors. In particular, the $16 \%$ difference on the secondary amplitude between Hilditch et al. (1992) and Duerbeck \& Rucinski (2007), as well as the large difference in systemic velocity $\left(V_{\gamma}\right)$ is bothersome.

The occultation-primary model fitting, shown in Figure 3 with photometric parameters listed in Table 4, gives essentially the same shape of light curve as before. However, it can be seen to be quite the reverse of the transit-primary model in its relative luminosities and dimensions of the stars (the hotter and cooler stars, here with suffixes $h$ and $c$, are usually denoted 1 and 2 in light curve analysis). The corresponding parameters must involve a completely different physical interpretation, that presumably involves a history of interaction between the close components. It may be noted that the different derived luminosities in $B$ and $V$ for the two components in the occultation-primary model imply a smaller difference in temperatures than the transit-primary solution. The difference in colour $\Delta(B-V)$ is now 0.096, as compared with 0.438 found for the fitting of Table 1. Keeping in mind the approximate proportionality between colour and inverse temperature, the appropriate secondary mean surface temperature rises to around $200 \mathrm{~K}$ less than that of the primary. Corresponding temperature-dependent secondary parameters of the fitting were adjusted accordingly. The formal errors, listed in brackets, again correspond to lower limits. The occultation solutions in both colours remain determinate for five parameters, but if we take the intercorrelations into account the error estimates rise by up to a factor of 10 , particularly in $B$.

It can be noted from Table 4 that the sum of the derived mean radii is slightly less than the critical condition $r_{1}+r_{2} \approx 0.75$ corresponding to the 'contact' condition for centrally condensed ('Roche') stellar models (Kopal 1959, Ch. 3). Hence, any contact between the photospheres of these stars, if present, should be marginal.

In Figure 4, we show the distribution of differences of measured flux and the photometric model. There appears a systematic trend in the phase range $\sim 0-50$ deg that could be interpreted as the eclipse of an area of enhanced relative brightness. The applied model only accounts only for standard effects, so there should be a downward blip 

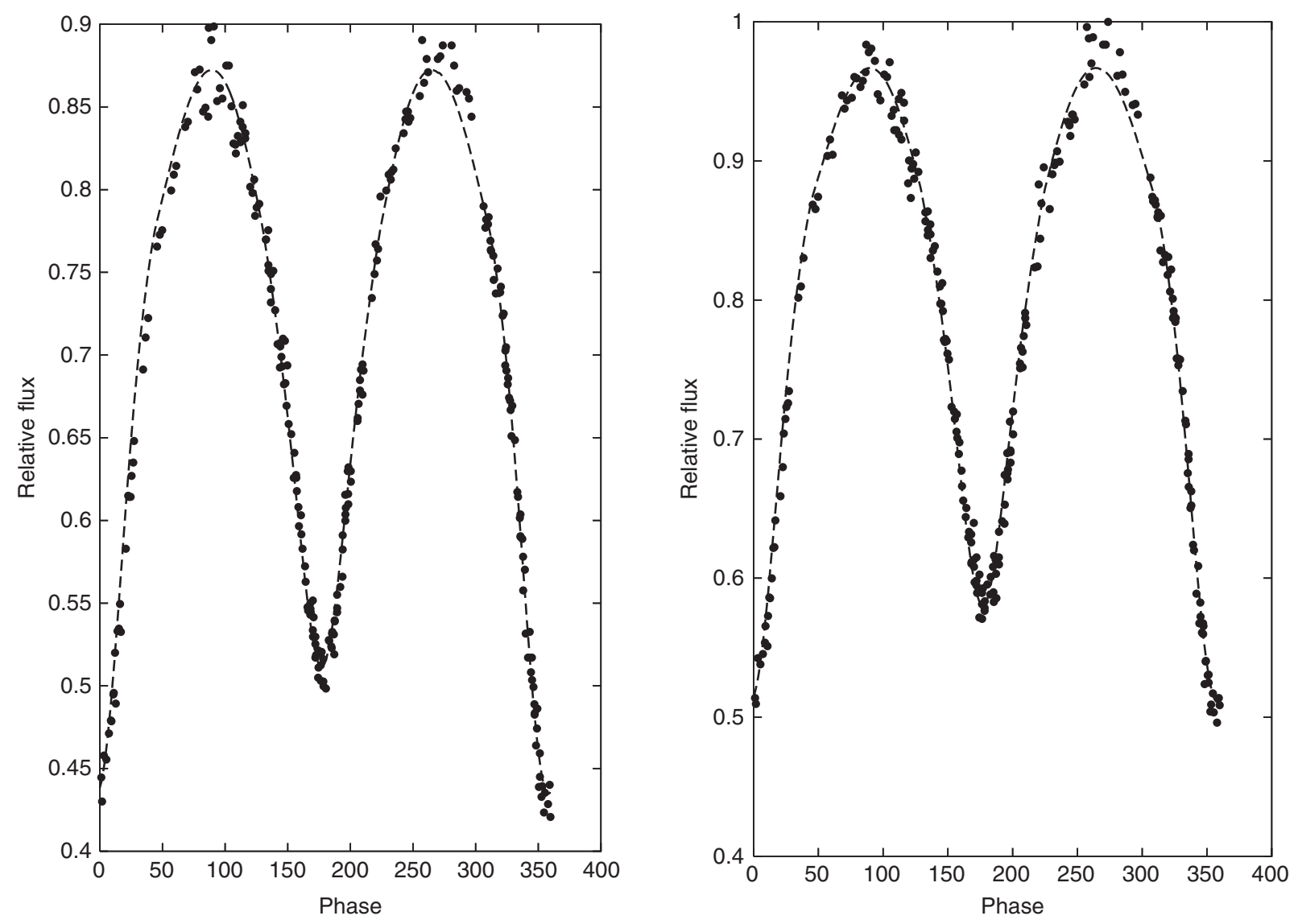

Figure 3 Light curves of RW Dor and their occultation-model fitting according to the parameters of Table 4 ( $B$ on left).

Table 4. RW Dor: primary occultation model light-curve fitting

\begin{tabular}{lc}
\hline Parameters & Values \\
\hline$T_{\mathrm{h}}(\mathrm{K})$ & 4800 \\
$T_{\mathrm{c}}(\mathrm{K})$ & 4600 \\
$M_{\mathrm{h}} / M_{\mathrm{c}}$ & 0.7 \\
$L_{\mathrm{h}}-\mathrm{B}$ & $0.392(6)$ \\
$L_{\mathrm{h}}-\mathrm{V}$ & $0.371(6)$ \\
$L_{\mathrm{c}}-\mathrm{B}$ & $0.608(6)$ \\
$L_{\mathrm{c}}-\mathrm{V}$ & $0.629(6)$ \\
$r_{\mathrm{h}}($ mean $)$ & $0.320(2)$ \\
$r_{\mathrm{c}}($ mean $)$ & $0.428(3)$ \\
$i($ deg $)$ & $76.9(0.2)$ \\
$\Delta \phi_{0}($ deg $)$ & 3.0 \\
$\Delta l$ & 0.015 \\
\hline
\end{tabular}

on the difference curve applying to the small range of phases when the bright spot is eclipsed. (A comparable effect was demonstrated, but for a dark spot producing an upward blip, in Budding \& Demircan 2007, p 360.) In turn, this could be related to a consequence of inter-component mass-transfer, since impinging, energy-releasing matter would tend to be diverted to the primary's receding hemisphere through the Coriolis action. The depth of the feature is almost the same in the two colours, indicating that any region of enhanced brightness would not be at a very much higher temperature than the surrounding photosphere. The argument is not very convincing on its own, as there appears a (smaller) continuation of the depression beyond the eclipse. This might be explained in terms of decreased apparent brightness as the spot recedes towards the retreating limb, though it would be more prudent not to put too much weight on the photometric evidence alone. Taken with the other aspects of the discussion, however, the downward blip in Figure 4 is consistent with a mass-transferring scenario.

\subsection{Times of Minimum}

In this connection, we may note the phase shift of around $3 \mathrm{deg}$ (Table 2) found for both the AO light curves and that of the HIPPARCOS satellite, corresponding to a mean epoch of about 1991.6. These phase shifts suggest a period decrease, even though there appears a lack of clear drift above $0.01 \mathrm{~d}$ in the rather sparse collection of $\mathrm{O}-\mathrm{C}$ data shown in the Atlas of Kreiner, Kim \& Nha (2001). In a simple conservative regime, accounting only for the orbital component of angular momentum, the period variation $\triangle P / P$ corresponds to a mass change $3 \Delta M / M_{l} \times\left(M_{g}-M_{l}\right) / M_{g}$, where $M_{g}$, the gainer, receives mass from $M_{l}$ the loser. Here $M_{l}$ should be the cooler, more massive star ( $=M_{1}$ in Table 3$)$ to produce a period decrease. Although this formula allows ideas about the order-of-magnitude of relevant quantities, the real situation becomes more complex when we add in 

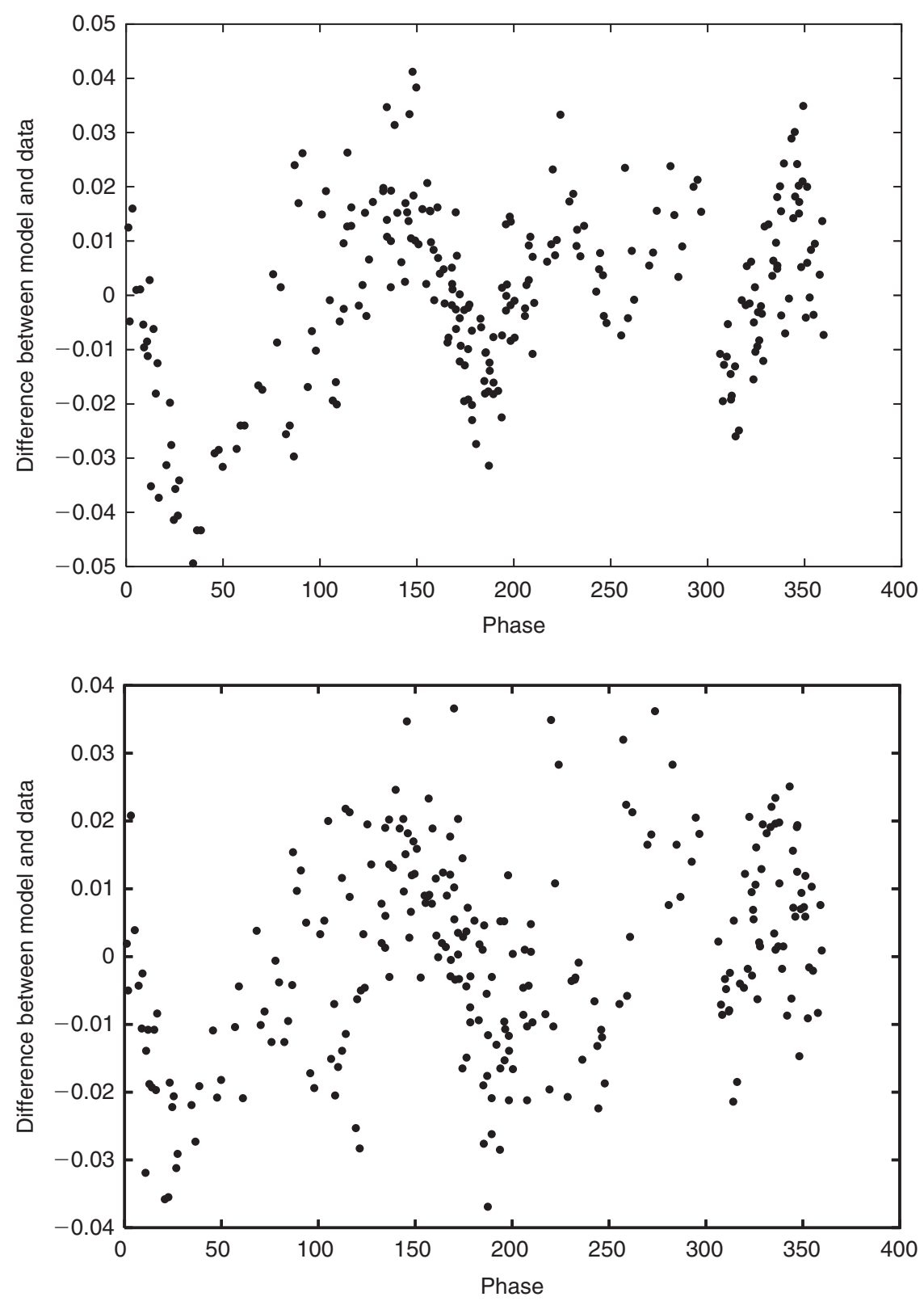

Figure 4 The distribution of differences between model and observed flux levels shows some gross systematic effect in the phase range $\sim 0-50 \mathrm{deg}$. This is interpreted as the eclipse of an area of enhanced relative brightness during that phase range.

the (non-negligible) rotational terms and take into account the way the gainer may respond to the inflowing material (e.g. Hazlehurst et al. 1977).

A light curve of RW Dor was obtained on April 20, 2006, using the $60 \mathrm{~cm}$ Optical Craftsman Telescope of Mt John University Observatory, equipped with its AAVSO courtesy CCD camera. The primary minimum was observed to occur at about $9 \mathrm{~h} 45 \mathrm{~m}$ UT, so that a relevant reference epoch was some 80245 revolutions after the epoch given in Equation (1), or at JD 2453845.64529. This reference was used in calculating observed phases. The next primary minimum should then have occurred at JD 2453845.93075, but, from binary model curve-fitting, the minimum was found to occur some $0.0249 \mathrm{~d}$ (phase shift of $31.4 \mathrm{deg}$ ) before this time, at JD 2453845.9059 (Epoch 80245.9129; see Table 5). The small heliocentric correction $-0.00023 \mathrm{~d}$, would put the observed time close to the above-mentioned eye estimate, but this correction, unlike the discrepancy, is small compared to the timing accuracy. This time of minimum discrepancy may be a continuation of the rather stronger negative trend shown in the last few timings in the Atlas of Kreiner et al., but displaced by another 20000 light cycles.

In order to check this point, more recent minima were timed at Mt Tarana Observatory, Bathurst, NSW in December 2006. The observer (CB) carried out CCD based aperture photometry with target, check and comparison stars exposed together. The data $\mathrm{S} / \mathrm{N}$ ratio was well in excess of 100 , so the internal precision of each measurement is estimated to be better than 0.005 mag. The light curve was sampled at 1.5 to 2 min intervals, with times synchronised manually to EAST/UT, rendering the 
Table 5. Times of primary minima (heliocentric JD 245..+) for RW Dor in 2006

\begin{tabular}{lcccccc}
\hline Date & $\begin{array}{c}\text { Obs. } \\
\text { KVW })\end{array}$ & $\begin{array}{c}\text { Formal } \\
\text { error }\end{array}$ & $\begin{array}{c}\text { Obs. } \\
\text { (curve-fit) }\end{array}$ & $\begin{array}{c}\text { Formal } \\
\text { error }\end{array}$ & $\begin{array}{c}\text { Calc. } \\
\text { Eq. (1) }\end{array}$ & $\begin{array}{c}\text { O-C } \\
\text { curve-fit }\end{array}$ \\
\hline Apr 20 & - & - & 3845.90590 & 0.00044 & 3845.93075 & -0.02485 \\
Dec 18 & 4087.97833 & 0.00009 & 4087.97853 & 0.00083 & 4088.00406 & -0.02553 \\
Dec 21 & 4091.11889 & 0.00017 & 4091.11897 & 0.00126 & 4091.14418 & -0.02521 \\
Dec 25 & 4095.11496 & 0.00010 & 4095.11585 & 0.00082 & 4095.14067 & -0.02482 \\
\hline
\end{tabular}

Heliocentric corrections were calculated using the java application at http://www.physics.sfasu. edu/astro/javascript/hjd.html

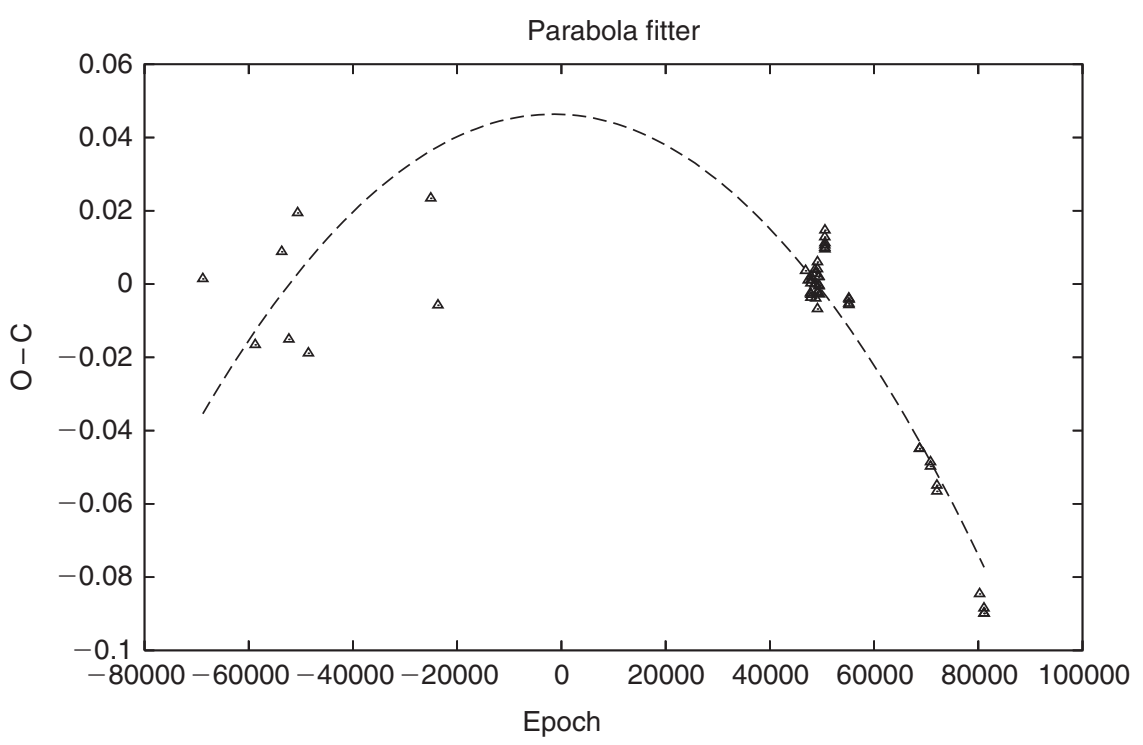

Figure 5 A parabola is fitted to 51 'observed minus calculated' $(\mathrm{O}-\mathrm{C})$ times of minimum data versus epoch number $(E)$ for RW Dor. The general conformity confirms a trend to period decrease, but the more accurate recent timings do not fit the mean parabola as well as might be expected if the period variation is constant.

timing of data probably accurate to $10^{-5} \mathrm{~d}$. The check star was GSC 9162 379, while HD $269301(B-V=0.379)$ was the main comparison. The possible photometric reference HD 35293 (see above) was not used, due to crowded field considerations. Observing conditions were generally good. Three minima were timed using the PERANSO software $^{2}$, which can implement both the well-known Kwee \& van Woerden (1956) (KVW) method and also a polynomial curve-fitting to the light curve, of up to 10th order. The light curve regions isolated for the timing were the primary minima $\pm \sim 0.8$ hours. Results are shown in Table 5 .

The epochs for the three new times of minimum, using Equation (1), are 81093.9098, 81104.9114 and 81118.9100, using the KVW times, and 81093.9105, 81104.9117 and 81118.9131 , using the polynomial fits. The trend towards period decrease is clearly maintained. In view of this, we carried out an analysis of 51 times of minima available from the Kreiner et al. database together with the new data, fitting the $\mathrm{O}-\mathrm{C}$ values to the form,

$$
\mathrm{O}-\mathrm{C}=a E^{2}+b E+c
$$

\footnotetext{
${ }^{2}$ http: //www. cbabelgium.com
}

(e.g. Budding et al. 2005). The derived optimal values are $a=-1.8 \pm 0.05 \times 10^{-11}, b=-5.6 \pm 2.4 \times 10^{-8}$, $c=0.046 \pm 0.001$ (Figure 5). The term $a$ gives the period change in days per unit cycle, yielding a mean rate of period decrease $\triangle P / P$ of about $-6.3 \times 10^{-11}$. From the foregoing formula we find an indicative rate of mass transfer of about $6.7 \times 10^{-8} \mathrm{M}_{\odot} \mathrm{y}^{-1}$ or $4.2 \times 10^{18} \mathrm{gm} \mathrm{s}^{-1}$. It should be noted that even though these minimum timings spread over more than a century the general coverage is sparse and uneven. The derived results are then rather a crude average, while it is also commonly found that period variations, when judged by a few observations only, may appear non-uniform, especially for W UMa-type binaries (Yamasaki 1975).

With a near-contact configuration, we should not expect velocities in a mass-transferring stream to be greatly in excess of thermal, i.e. $\sim 10^{6} \mathrm{~cm} \mathrm{~s}^{-1}$ (Lubow \& Shu 1975), making a total available energy $f$, say, of less than $\sim 10^{30} \mathrm{erg} \mathrm{s}^{-1}$ or less than $1 \%$ of the system's total radiated energy $(L)$. However, the projected size $\left(\pi r^{2}\right.$, say) of the region of interaction need only be a few percent of the total photosphere $\left(4 \pi R^{2}\right.$, say), to produce a significant local temperature rise. Thus, as a fraction of the average 
surface flux $F$, the flux in the interaction region $F^{\prime}$ is of order

$$
F^{\prime} \sim\left(\frac{4 R^{2} f}{r^{2} L}\right) F .
$$

Given the relatively cool surface temperatures of the stars in RW Dor, a local temperature increase will have a relatively strong effect in the $B$ and $V$ optical ranges. From Figure 4 it can be seen that the photometric irregularity is less than $5 \%$ of the system's light, so not inconsistent with the formation of such a local 'hot spot', eclipsed during the emergence from primary minimum phases.

\section{Discussion}

The problem of overabundance of contact binary systems was recently revived by Paczyński et al. (2006) and has been commented on by Eker et al. (2006). Budding (1984) considered a variant of the Case-B mass-transfer scenario, widely believed to apply to classical Algols, in which the gainer is originally a low-mass companion less than $\sim 0.2$ times the original primary mass and at a distance of order 10 times its radius. For plausible rates of accretion within the gainer's Roche lobe, there is then a comfortably long period $\left(\sim 10^{9} \mathrm{y}\right)$ of close to contact status, in which the system appears as a W-type overcontact configuration, with masses of the loser, expressed as a fraction of the system, typically in the range $\sim 0.6-0.4$, and a moderate degree of angular momentum loss provided by various alternative possible braking mechanisms, that inhibit the formation of a classical Algol binary of low mass. In this connection, we note also the inference of great age to the binary coming from Hilditch et al. (1992). Although one might expect mass-transfer rates in the approach phase of typical Case-B scenarios to be rather larger than the indicative rate given above, there were sufficient control parameters affecting the mass and angular momentum redistribution in Budding's scenario to allow the possibility of its relevance to RW Dor, if mass really is being transferred from the presently more massive star to its hotter companion.

The issues of slow period decrease and overluminosity of the smaller component, as observed in RW Dor or other W-type contact binaries, are natural consequences of this picture. The model predicts that as many as $10 \%$ of all stars of mass greater than $1.5 \mathrm{M}_{\odot}$ should be initially accompanied by low-mass companions (less than $0.2 \mathrm{M}_{\odot}$ ). This point is receiving increased support from recent more detailed studies of binary mass ratio distribution (e.g. Kouwenhoven et al. 2005). The model also predicts the end point of such evolution to be a dwarf nova, when the erstwhile primary has reduced to a white dwarf and the relatively nearby, still relatively low-mass companion itself starts to approach its surrounding Roche lobe. This is reminiscent of the original scenario of Kraft (1967), although not consistent with more recent contact binary evolution schemes (e.g. Webbink 1979; Vilhu 1981).

An alternative configuration would make the secondary, originally quite more massive than the present total mass of the system, the present loser. The primary then becomes a generally MS-like star. The hotter region of inflow should then become exposed in the phase range just after primary minimum. Apart from the difficulties of reconciling this scenario with the observed light-curve distortion and the trend to period decrease, a complex prior history of interaction with considerable systemic mass and angular momentum loss is called for. The present state of general understanding of contact and near contact binaries still allows for such possibilities, however, given the range of options that could affect angular momentum loss.

The role of magnetic field related braking mechanisms is often considered in this context (e.g. Eker et al. 2006). It is true that many contact binaries show photometric irregularities that could be associated with surface maculation effects. There are also optical and UV emissions, usually associated with chromospheric activity (Barden 1985; Vilhu \& Walter 1987). However, X-ray and radio emissions do not scale up with rotation speed for contact binaries in the same way as one would expect from comparable variations in RS CVn-type binaries (Rucinski \& Seaquist 1988; Vilhu, Caillault \& Heise 1988). An interpretation put on this is that differential rotation, a prerequisite for strong magnetic field generation in $\alpha-\omega$-type field theories, becomes inhibited as binary components approach contact (Beasley et al. 1993).

Magnetic cycles may introduce a quasi-sinusoidal term into the longer term trend of period variation (e.g. Applegate 1992; Van't Veer 1993; Budding \& Demircan 2007, Ch. 8). With RW Dor the change of phase of primary minimum observed in 2006 seemed, at first, rather large, suggestive of a relatively large drop in period. Fitting of all times of minima, however, show the mean rate of period variation to be not extraordinary. Period changes in the binary derived for the more recent time bases still suggest some non-uniformity over shorter intervals, however, indicative of physical complexities in this binary.

\section{Conclusions}

We have presented and analysed original light curves of RW Dor, whose period is among the shortest known for regular eclipsing binaries, and compared these with that of the HIPPARCOS satellite and a more recent (2006) light curve taken at the Mt John University Observatory. Curve-fittings to all these data sets can produce agreement on parameter sets, but we note that photometry alone is very ambiguous in fixing physical conditions.

Hilditch et al. (1992), aware of this, deduced an occultation (W-type) contact binary configuration from a small number of radial velocity measurements to which a pair of sinusoids were fitted. We have introduced a more developed and physically flexible fitting function to take full account of proximity or other effects on the measured radial velocities and added a few more recently obtained radial velocity values, although the configuration found by Hilditch et al. is still upheld with little modification.

Our review of times of minima show a general trend to period decrease, although more recent timings 
may allow a faster rate than the long term average. A suggested mass transfer of about $7 \times 10^{-8} \mathrm{M}_{\odot} \mathrm{y}^{-1}$ is found plausible when set against the observed light curve irregularity and consistent with the initially lowmass companion, Case-B scenario of Budding (1984) that reconciles some outstanding puzzles of the W UMa configuration. However, alternative scenarios cannot be ruled out. More continuous detailed observations, including more spectroscopy, is desirable to check these latter ideas.

\section{Accessory Publication}

An Accessory Publication listing the raw data, with dates times and calibrated $V$ and $B-V$ magnitude and colours, and the heliocentric corrections is available from the author or, until December 2012, Publications of the Astronomical Society of Australia.

\section{Acknowledgement}

The observations made with the $1.8 \mathrm{~m}$ telescope at Mt Stromlo in the late 1980s (referred to in Section 2) were supported by Carter Observatory, and, particularly, by Dr Denis J. Sullivan, of the Physics Department, Victoria University of Wellington, who provided technical help and suggestions as well as solving various logistic problems connected with making the observations and their subsequent reduction. Practical help from staff at the Mt John University Observatory, and also Volkan Bakış, during the 2006 observations there is duly recognized. The data on the 51 times of minima discussed in section 3.1 were kindly assembled by Prof. O. Demircan of Çanakkale Onsekiz Mart University, Turkey, who also made useful comments on an earlier version of this paper. Two unnamed referees provided extensive comments on an earlier version of this paper, which have undoubtedly improved the final result.

\section{References}

Applegate, J., 1992, ApJ, 385, 621

Banks, T. \& Budding, E., 1990, Ap\&SS, 167, 221
Banks, T., Sullivan, D. J. \& Budding, E., 1990, Ap\&SS, 173, 77

Barden, S. C., 1985, ApJ, 295, 162

Beasley, A. J., Ball, L. T., Budding, E., Slee, O. B. \& Stewart, R. T., 1993, AJ, 106, 1656

Budding, E., 1981, in Investigating the Universe, Ed. Kahn, F. D. (Dordrecht: Riedel), 271

Budding, E., 1984, A\&A, 130, 324

Budding, E., Bakiş, V., Erdem, A., Demircan, O., Iliev, L., Iliev, I. \& Slee, O. B., 2005, Ap\&SS, 296, 371

Budding, E. \& Demircan, O., 2007, Introduction to Astronomical Photometry 2nd edn (Cambridge: Cambridge University Press)

Duerbeck, H. \& Rucinski, S., 2007, AJ, 133, 169

Eker, Z., Demircan, O., Bilir, S. \& Karataş, Y., 2006, MNRAS, 373 1483

ESA, 1997, The Hipparcos and Tycho Catalogues (Noordwijk: ESTEC), $1548 \mathrm{pp}$

Hazlehurst, J., Refsdal, S. \& Stobbe, C., 1977, A\&A, 58, 47

Hertzsprung, E., 1928, BAN, 4, 154

Hilditch, R. W., 1989, SSRv, 50, 289

Hilditch, R. W., Hill, G. \& Bell, S. A., 1992, MNRAS, 225, 285

Kaluzny, J. \& Caillault, J.-P., 1989, AcA, 39, 27

Kholopov, P. N., 1985, General Catalogue of Variable Stars (Moscow: Nauka Publ.)

Kopal, Z., 1959, Close Binary Systems (London: Chapman \& Hall) Kopal, Z., 1979, ASSL, 77

Kouwenhoven, M. B. N., Brown, A. G. A., Zinnecker, H., Kaper, L. \& Portegies Zwart, S. F., 2005, A\&A, 430, 137

Kraft, R., 1967, ApJ, 79, 395

Kreiner, J. M., Kim, C.-H. \& Nha, I.-S., 2001, An Atlas of OC Diagrams of Eclipsing Binary Stars (Krakow: Wydawnistwo Naukowo)

Kwee, K. K. \& van Woerden, H., 1956, BAN, 12, 327

Lubow, S. H. \& Shu, F. H., 1975, ApJ, 198, 383

Marton, S. F., Grieco, A. \& Sistero, R. F., 1989, MNRAS, 240, 931

McLaughlin, D. B., 1927, AJ, 38, 45

Paczyński, B., Szczygiel, D. M., Pilecki, B. \& Pomanski, G., 2006, MNRAS, 368, 1311

Rucinski, S. M. \& Seaquist, E. R., 1988, AJ, 95, 1837

Rucinski, S. M. \& Lu, W., 1999, AJ, 118, 2451

Van't Veer, F., 1993, in New Frontiers in Binary Star Research, Eds. Leung, K. C. \& Nha, I. S. (San Francisco: ASP), 229

Vilhu, O., 1981, Ap\&SS, 78, 401

Vilhu, O. \& Walter, F. M., 1987, ApJ, 321, 958

Vilhu, O., Caillault, J.-P. \& Heise, J., 1988, ApJ, 330, 922

Webbink, R., 1979, ApJ, 227, 178

Wilson, R. E. \& Devinney, E. J., 1971, ApJ, 166, 605

Yamasaki, A., 1975, Ap\&SS, 34, 413 